\title{
En deçà du bien et du mal écrire. Pour une saisie en temps réel des invariants opérationnels de l'écriture
}

\section{Christophe Leblay}

\section{(2) OpenEdition \\ Journals}

Édition électronique

URL : http://journals.openedition.org/pratiques/1430

DOI : $10.4000 /$ pratiques. 1430

ISSN : 2425-2042

Éditeur

Centre de recherche sur les médiations (CREM)

Édition imprimée

Date de publication : 15 décembre 2009

Pagination : 153-167

Référence électronique

Christophe Leblay, "En deçà du bien et du mal écrire. Pour une saisie en temps réel des invariants opérationnels de l'écriture », Pratiques [En ligne], 143-144 | 2009, mis en ligne le 19 juin 2014, consulté le 01 mai 2019. URL : http://journals.openedition.org/pratiques/1430; DOI : 10.4000/pratiques.1430 


\section{En deçà du bien et du mal écrire. Pour une saisie en temps réel des invariants opérationnels de l'écriture}

\section{Christophe Leblay}

Soveltavan kielentutkimuksen keskus, Jyväskylän yliopisto, Finlande

Institut des Textes et Manuscrits Modernes (UMR 8132 CNRS/ENS), Paris GDR - CNRS 2657 « Approche Pluridisciplinaire de la Production Verbale Ecrite » \& COST Action ISO703 « European Research Network on Learning to Write Effectively »

Dans ce travail, sera développé, à partir de la génétique du texte, un point de vue selon lequel la notion d'invariant opérationnel, ou processuel, permet une mise en relief de 1'expertise. Il s'agira alors, principalement, de saisir un avant-texte numérisé, au moyen de l'enregistrement tant des événements d'écriture que des opérations d'écriture ${ }^{(1)}$.

Il s'agit de transposer, et non seulement d'appliquer, une méthodologie (et pas nécessairement les concepts qui vont avec) à une didactique des langues étrangère, maternelle et seconde. Ainsi, plus particulièrement, sera transposée la méthodologie linguistique de la critique génétique à la didactique de la production verbale écrite (PVE) et plus précisément à la didactique de la PVE en français langue étrangère dans un contexte finnophone.

L'un des principaux acquis de la génétique du texte est la mise en évidence de modèles de transcriptions qui peuvent rendre compte des événements et des opérations d'écriture. Transposées à des techniques d'enregistrement de la production en temps réel, ces opérations se retrouvent associées, en un contexte dynamique, aux mouvements du curseur.

Le rôle du déjà écrit se présente alors comme un facteur déterminant : il permet de mettre en parallèle les opérations qui s'inscrivent à la suite du déjà écrit et les opérations d'écriture qui font retour sur le déjà écrit. Il devient alors possible de comparer des productions expertes à des productions novices, dans le contexte FLE qui est le nôtre, afin de mieux saisir ce qu'est l'écriture expérimentée en contexte dynamique.

(1) Voir le numéro 155 de Langue française (Septembre 2007) : Avant le texte : les traces de l'élaboration textuelle (Edité par Lucile Chanquoy et Irène Fenoglio). 


\section{Corpus de PVE en temps réel}

L'idée très simple est de montrer qu'un corpus de production écrite peut apparaître dans une forme notée en heures, en minutes, en secondes et non pas, systématiquement, en nombre de lignes et de pages écrites. Particularité qui progressivement tend à s'étendre davantage au sein des études en PVE et en didactique de la production écrite en langues maternelle, étrangère et seconde.

\subsection{Productions orale et écrite}

Pour les études consacrées à la production écrite, il s'agit surtout de ne plus oublier ce double mouvement : tout d'abord, celui d'enregistrer parce que la production écrite, a contrario du « produit » de l'écriture, reste, à l'instar de celle de l'oral, par nature, fugitive (il s'agit alors de laisser le mouvement, la dynamique de production s'exprimer) ; ensuite, celui de stabiliser les traces de ce mouvement par des procédés de linéarisation et de transcription.

Sans chercher à prolonger cette analogie entre corpus écrits en temps réel et corpus oraux, il est nécessaire néanmoins de s'arrêter quelques instants pour insister sur l'idée que la prise en compte du temps réel en production écrite redonne aux études de la production écrite leur véritable statut d'études de production. Peut-on parler de production écrite en ne considérant que le (texte) produit, comme, trop souvent encore, on peut l'entendre dire ? Peut-on saisir la production (écrite) autrement qu'en temps réel ? Il semble y avoir donc déjà, en aval, nécessité d'une remise à plat des concepts utilisés pour étudier une production écrite ${ }^{(2)}$. L'essentiel est de pouvoir être capable d'appréhender les unes et les autres dans leur relation au temps. Pour reprendre une expression de Claudine Fabre-Cols, l'écriture est « fondamentalement dans le temps» $(2004,21)$.

\subsection{Enregistrer une production écrite spontanée}

L'acte fondateur, dans ce genre d'études, est d'enregistrer l'écriture de façon à pouvoir replacer l'écriture dans sa relation au temps ${ }^{(3)}$. Et l'enregistrement ne fait rien d'autre que de garder la trace scripturale dans son inscription temporelle. Il existe sur le marché un certain nombre de logiciels, dit de temps réel, qui sont de fait des logiciels d'enregistrement ${ }^{(4)}$. Pour le présent travail, c'est à partir du logiciel ScriptLog que nous avons pu enregistrer dix scripteurs en temps limité.

La tâche choisie relève de la spontanéité, de l'inachevé. Elle cherche à prendre le scripteur sur le vif, au sein de son inquiétude : saisir un état de ce qui est en mouvement. Elle est, en cela, limitée à 15 minutes; elle cherche à prendre un simple échantillonnage de la production de chaque scripteur ${ }^{(5)}$.

(2) Pour s'en convaincre, il suffit de prendre un document comme celui du Cadre Européen Commun de Référence (CECR), où les termes de production écrite réfèrent de fait au produit.

(3) Claire Blanche-Benveniste soulignait déjà (2000), en citant M. A. K. Halliday, que les études de l'oral ont pris véritablement leur essor à partir du moment où l'enregistrement de l'oral fut possible.

(4) Pour se limiter aux plus utilisés : Eye and Pen (www.eyeandpen.net.), Inputlog (www.inputlog.net), JEdit (www.nada.kth.se/iplab/trace-il/index.html) et ScriptLog (www.scriptlog.net).

(5) L'idée est que ce qui se passe en un temps restreint est représentatif de ce qui se passe sur un temps plus long. La spontanéité de la production a, selon une définition psychologique, le sens très général d'une phase au cours de laquelle n'intervient aucun acte conscient. Il n'entre pas dans le cadre de ce travail d'interroger le rapport entre génétique du texte et 
Le choix de deux groupes de cinq scripteurs par compétence (novice $v s$ experte) semble, dans un premier temps, être suffisant pour pouvoir mettre en place certaines caractéristiques minimales par compétence. Il fallait trouver une tâche de production qui puisse permettre à chacun des deux groupes de s'exprimer librement sans avoir à forcer sa compétence ni vers le haut, ni vers le bas. Une telle tâche devait permettre aux scripteurs novices (groupe 1) de pouvoir s'exprimer sans redouter que trop leur soit demandé, ni même aux scripteurs experts (groupe 2) de pouvoir s'exprimer sans une quelconque sous-estimation de leur niveau. La tâche permet donc aux deux groupes de s'exprimer, conformément à leurs compétences, mais aussi conformément à leurs expériences du monde : il était naturel de partir de l'idée que des apprentis scripteurs, parce que, généralement plus jeunes, pouvait avoir du monde une expérience plus limitée que des scripteurs experts, généralement plus âgés, et donc plus expérimentés. D'où une tâche faisant appel soit à l'imagination, soit à l'expérience.

La consigne a été la suivante ${ }^{(6)}$ :

Décrivez votre environnement idéal ainsi que les personnes ou les êtres qui pourraient y vivre. Imaginez-le sans vous limiter.

Vous pouvez écrire à la première personne (Je), ou à la deuxième (Tu), ou bien encore à la troisième (Il/Elle).

Mais, c'est seulement après avoir pu enregistrer le déroulement de l'écriture que commence véritablement l'intérêt de ce genre de matériel. D'une manière variée, ces logiciels offrent, non seulement la possibilité de conserver les traces exhaustives de l'écriture, mais produisent un ensemble de documents permettant un début d'analyse des processus scripturaux.

\section{Visualisation du processus d'écriture}

A se contenter de faire seulement mention du (ou des) processus d'écriture, on en vient facilement à oublier ce qu'une telle notion peut recouvrir. Regardons, très concrètement, quels types de documents nous sont proposés pour appréhender, avec un nouveau regard, des notions peu exploitées, mais élémentaires, comme celle d'événement d'écriture.

\subsection{Le fichier d'enregistrement des événements}

Le document élémentaire, nommé en anglais Log, que les logiciels de temps réel mettent à disposition, est, selon les propres mots de Strömqvist (2006a, 46), « un fichier numérique contenant une représentation exhaustive des événements surgis pendant l'activité d'écriture avec leur distribution temporelle ». L'événement d'écriture est alors présenté comme une unité élémentaire en production écrite. A chaque fois qu'un scripteur frappe le clavier, il se produit un événe-

psychanalyse ni même de mettre en contraste certains points de dissonance entre la critique génétique et la psycholinguistique. Bien au contraire, il s'agit, dans ces pages, de prendre le parti que l'une et l'autre de ces disciplines peuvent apporter, en se conjuguant, une réponse pertinente à qui s'interroge sur l'écriture.

(6) La consigne a été donnée en langue maternelle, soit en finnois : Millainen voisi olla ihanteellinen elinpaikka ja -ympäristö sekä siihen mahdollisesti kuuluvat muut ihmiset/olennot, jos toive olisi toteutettavissa ilman minkäänlaisia rajoituksia?

Voit kirjoittaa tekstin valintasi mukaan joko yksikön ensimmäisessä (minä), yksikön toisessa (sinä) tai yksikön kolmannessa (hän) persoonassa. 
ment ${ }^{(7)}$. Ce fichier (figure 1) des événements reste un document riche mais néanmoins encombré : il se présente, le plus souvent, en une série de colonnes dont la première, située à gauche, est celle du temps. Elle est celle à partir de laquelle le fichier prend sens, puisqu'elle montre le temps écoulé (en minutes, secondes et millièmes de secondes) depuis le début de la tâche d'écriture. Dans la deuxième colonne, le fichier prend note du type d'événement concerné : le type correspond à la frappe d'une minuscule, tandis que le type 5, par exemple, correspond à la frappe d'un effacement, et ainsi de suite. Les troisième et quatrième colonnes notent la position de la frappe à l'écran en s'attachant à distinguer la position de départ (colonne trois) de celle d'arrivée (colonne quatre). Enfin la cinquième colonne note l'événement par excellence : elle donne de fait la succession des événements et doit se lire verticalement. L'écriture, dans son déroulement temporel, est alors notée d'une manière exhaustive : tout est donné.

Il est vain de donner en entier un fichier d'enregistrement d'une production de 15 minutes puisque celui peut représenter jusqu'à 50 pages (Time New Roman, 12). Voici, à titre d'exemple, les 48 premières secondes de production qui correspondent à l'enregistrement du texte produit (4a) et à sa transcription (4b).

$\begin{array}{llllllllll}\text { time } & \text { type } & \text { from } & \text { to } & \text { key } & \text { time } & \text { type } & \text { from } & \text { to } & k e y \\ 0.00 & 10 & 1 & 0 & <\text { START }> & 28.46 & 7 & 30 & 30 & \mathrm{~d} \\ 5.75 & 7 & 0 & 0 & \mathrm{E} & 28.65 & 7 & 31 & 31 & \mathrm{e} \\ 6.68 & 7 & 1 & 1 & \mathrm{n} & 28.75 & 7 & 32 & 32 & \\ 7.20 & 7 & 2 & 2 & \mathrm{v} & 28.91 & 7 & 33 & 33 & \mathrm{v} \\ 7.91 & 7 & 3 & 3 & \mathrm{i} & 29.03 & 7 & 34 & 34 & \mathrm{e} \\ 8.08 & 7 & 4 & 4 & \mathrm{r} & 29.28 & 7 & 35 & 35 & \mathrm{r} \\ 8.20 & 7 & 5 & 5 & \mathrm{o} & 29.56 & 7 & 36 & 36 & \mathrm{~d} \\ 8.45 & 7 & 6 & 6 & \mathrm{n} & 29.68 & 7 & 37 & 37 & \mathrm{u} \\ 8.60 & 7 & 7 & 7 & \mathrm{n} & 29.98 & 7 & 38 & 38 & \mathrm{r} \\ 8.73 & 7 & 8 & 8 & \mathrm{a} & 30.05 & 7 & 39 & 39 & \mathrm{e} \\ 10.06 & 5 & 9 & 9 & <\text { DELETE }> & 34.51 & 8 & 40 & 40 & <\text { LEFT }> \\ 10.63 & 7 & 8 & 8 & \mathrm{e} & 35.00 & 8 & 39 & 39 & <\text { LEFT }> \\ 10.80 & 7 & 9 & 9 & \mathrm{~m} & 35.18 & 8 & 38 & 38 & <\text { LEFT }> \\ 10.90 & 7 & 10 & 10 & \mathrm{e} & 35.35 & 8 & 37 & 37 & <\text { LEFT }> \\ 11.01 & 7 & 11 & 11 & \mathrm{n} & 35.50 & 8 & 36 & 36 & <\text { LEFT }> \\ 11.21 & 7 & 12 & 12 & \mathrm{t} & 35.66 & 8 & 35 & 35 & <\text { LEFT }> \\ 11.45 & 7 & 13 & 13 & & 35.83 & 8 & 34 & 34 & <\text { LEFT }> \\ 11.68 & 7 & 14 & 14 & \mathrm{i} & 36.00 & 8 & 33 & 33 & <\text { LEFT }> \\ 11.95 & 7 & 15 & 15 & \mathrm{~d} & 36.16 & 8 & 32 & 32 & <\text { LEFT }> \\ 12.31 & 7 & 16 & 16 & \mathrm{e} & 36.33 & 8 & 31 & 31 & <\text { LEFT }> \\ 12.71 & 7 & 17 & 17 & \mathrm{a} & 36.51 & 8 & 30 & 30 & <\text { LEFT }> \\ 12.78 & 7 & 18 & 18 & \mathrm{l} & 36.68 & 8 & 29 & 29 & <\text { LEFT }> \\ 21.46 & 7 & 19 & 19 & <\text { CR }> & 36.85 & 8 & 28 & 28 & <\text { LEFT }> \\ 22.75 & 7 & 20 & 20 & <\text { CR> } & 37.01 & 8 & 27 & 27 & <\text { LEFT }> \\ 26.28 & 7 & 21 & 21 & \mathrm{~B} & 37.18 & 8 & 26 & 26 & <\text { LEFT> } \\ 26.71 & 7 & 22 & 22 & \mathrm{e} & 37.50 & 8 & 25 & 25 & <\text { LEFT }> \\ 26.88 & 7 & 23 & 23 & \mathrm{u} & 38.31 & 5 & 24 & 24 & <\text { DELETE }> \\ 27.00 & 7 & 24 & 24 & \mathrm{a} & 38.95 & 8 & 23 & 23 & <\text { RIGHT }> \\ 27.33 & 7 & 25 & 25 & \mathrm{c} & 41.01 & 7 & 24 & 24 & \mathrm{u} \\ 27.75 & 7 & 26 & 26 & \mathrm{o} & 43.91 & 8 & 25 & 25 & <\text { RIGHT }> \\ 27.88 & 7 & 27 & 27 & \mathrm{u} & 44.15 & 8 & 26 & 26 & <\text { RIGHT }> \\ 28.10 & 7 & 28 & 28 & \mathrm{p} & 44.31 & 8 & 27 & 27 & <\text { RIGHT }> \\ 28.26 & 7 & 29 & 29 & & 44.48 & 8 & 28 & 28 & <\text { RIGHT }>\end{array}$

(7) Et d'une manière générale, à chaque fois qu'un scripteur prend un crayon, ou touche un clavier tactile. La notion d'événement reste opérante indépendamment des outils d'écriture. 


$\begin{array}{llllllllll}\text { time } & \text { type } & \text { from } & \text { to } & \text { key } & \text { time } & \text { type } & \text { from } & \text { to } & k \text { key } \\ 44.68 & 8 & 29 & 29 & \text { < RIGHT }> & 45.71 & 8 & 35 & 35 & <\text { RIGHT }> \\ 44.85 & 8 & 30 & 30 & \text { <RIGHT }> & 45.88 & 8 & 36 & 36 & <\text { RIGHT }> \\ 45.01 & 8 & 31 & 31 & \text { <RIGHT }> & 46.08 & 8 & 37 & 37 & <\text { RIGHT }> \\ 45.18 & 8 & 32 & 32 & \text { <RIGHT }> & 46.31 & 8 & 38 & 38 & <\text { RIGHT }> \\ 45.36 & 8 & 33 & 33 & \text { <RIGHT }> & 46.76 & 8 & 39 & 39 & <\text { RIGHT }> \\ 45.53 & 8 & 34 & 34 & \text { <RIGHT }> & 47.66 & 7 & 40 & 40 & ,\end{array}$

Sans être aussi systématique que Strömqvist (2006a, 47 ; 2006b, 47), il est possible, à qui s'en donne la peine, de travailler à partir de ce genre de fichier exhaustif pour observer et analyser la production, mais la tâche est ingrate. Les logiciels offrent d'autres documents qui permettent une bien meilleure appréhension en proposant une amorce de linéarisation des événements d'écriture.

\subsection{La traduction linéarisée des événements}

S'appuyant sur le document précédent, la traduction linéarisée des événements, en anglais linear text, offre au lecteur une bien meilleure visualisation du processus d'écriture ${ }^{(8)}$. Est traduit sur la ligne ce qui était noté précédemment en colonne avec un encodage simplifié ( $c f$. figure 2) :

(2) $<$ START $>$ Environna $<$ DELETE $>$ ement idéal $<$ CR $><$ CR $>$ Beuacoup de verdure $<$ LEFT16 $><$ DELETE $><$ RIGHT $>$ u $<$ RIGHT15 $>$, peu de pollution, suffisamment d'espace pour que tuot $<$ DELETE3 $>$ out un chacun se trouve ' $l$ ' $i<$ DELETE4 $>$ à $l$ 'aise asv $<$ DELETE2 $>$ avec soi-m^me $<$ DELETE3 $>$ ême etv avec les autres $<$ CUT $>$ $<$ DELETE $><$ CUT $><$ DELETE $><$ CUT $>$. Le climat? Je dois dire que les saisons distinctes et variées typiques du Nord ma plaisent $<$ CUT $><$ DELETE $>$ e $<$ CUT $>$, etv que ce $<$ DELETE $>$ 'est vquelque chose que nje $<$ DELETE4 $>$ je voudrais avoir dans un unvironnement idéal. Do $<$ DELETE2 $>$ Autrement ndit, un été chaud et ensoileillé ave $<$ DELETE $>$ e $<$ DELETE3 $>$ sans oublier évidemment les journées lumineuses et etrêmement longues $<$ CUT $>\mathrm{x}<\mathrm{CUT}>{ }^{\prime}$ 'la $<$ DELETE3 $>$ comme en Finlande en été, un hiver blanc, avec du doleil et de la naige $<$ DELETE4 $>$ eid $<$ DELETE $>$ ge, et encore duex saisio $<$ DELETE2 $>$ ons de "transmission", de passage on peut dire, pour passer $\mathrm{du}$ froid au chaud ou läinverse $<$ DELETE9 $>$ inversemenrt $<$ DELETE2 $>\mathrm{t}$ : le prinp $<$ DELETE $2>$ ntemps et 1 'ai $<$ DELETE $>$ utomne. $<$ CR $><$ CR $>$ Je ne peux $<$ DELETE10 $>$ S'il n'est pas possible, pour moi, d'imaginer une vie idéale sans la nature éblouissante $<$ DELETE13 $>$ telle que je la connais dans mon pays natale, il n'äe $<$ DELETE3 $>$ 'est possible non plus pour $<$ DELETE $><$ CUT $>$ $<$ DELETE $>$ m $<$ CUT $>$ éga $<$ DELETE $3>$ tout aussi im $<$ CUT $><$ DELETE $14>$ e d'imaginetr $<$ DELETE2 $>$ r un evie heureuse sans la pårésence des enfants et des animaux. $<$ CUT $><$ DELETE $>$ e $<$ CUT $><$ DELETE $><$ CUT $>$ $<$ DELETE $>$ S $<$ CUT $><$ DELETE $><$ RIGHT $>$ U $<$ CUT, $<$ CUT $><$ DELETE $5>$ e existence $<$ CUT $><$ DELETE $><$ CUT $><$ DELETE $>(<$ DELETE2 $>$. Les enfants et les animaux font partie de la nature au sens $<$ DELETE $8>$. $<$ CUT $>$ Que me vionet $<$ DELETE11 $>$ 'est-ce qui me vient en tête spontanément quand on me donne la tâche d' $<$ DELETE $>$ e décrire un environnement idéal? $<$ CUT $><$ DELETE $><$ CUT $>$ $<$ CR $>$ Toute ma vie, jä'a $<$ DELETE3 $>$ ' $<$ DELETE16 $>$ J'ai toutjours vécu au bord de la mer. $<$ DELETE $>$ m $<$ DELETE $>$, ou plus précisément, je vis mes hivers au bord de 1 amer proprment $<$ DELETE4 $>$ ement dite et mes étés à 1 acampgne $<$ DELETE9 $>$ a campagne au bord d'un grand lac. Cette présence de l'eau dans mon environnement $\mathrm{q}<$ DELETE $>$ est quelque chose qui me manque lorsque je suis

(8) A titre de comparaison, voir les tentatives de linéarisation (et d'interprétation) proposées par Claire Doquet-Lacoste (2004a) à partir du logiciel Genèse du texte. 
Très rapidement il est possible de s'approprier cette traduction linéraire dans ses grandes lignes. Ainsi, $<$ DELETE2 $>$ note la suppression de l'événement précédent, le chiffre notant, quant à lui, le nombre d'événements supprimés. Cette traduction met aussi en évidence le déplacement du curseur : $<$ LEFT $>,<$ RIGHT $>$ notent l'utilisation de la flèche pour se déplacer dans le texte, en concurrence avec l'utilisation de la souris.

Il n'empêche : si cette traduction linéarisée des événements est très utile pour se représenter le processus d'écriture dans son ensemble, elle reste néanmoins peu lisible ; cette linéarisation est surtout remarquable parce qu'elle permet, à son tour, un accès à d'autres procédés de visualisation.

Au-delà du fichier d'enregistrement et de la traduction linéaire des événements d'écriture, il est possible de reconstituer une transcription encore plus lisible et bien plus exploitable. Si le premier document offre une liste chronologique des événements et le deuxième une linéarisation de ces mêmes événements, le troisième document, que nous nous proposons de présenter dans les pages qui suivent, offre une visualisation sous la forme de transcriptions.

\subsection{Transcriptions des événements}

Avant de donner à voir une possibilité de transcriptions de ces événements et des mouvements associés du curseur, il semble inévitable de prendre le temps de regarder ce que peuvent être des conventions de transcriptions pour le processus d'écriture. Ainsi, dans le cas de logiciels de type ScriptLog, pour ne citer que celui qui nous occupe, il existe un système informatisé d'analyse produisant des représentations très pertinentes mettant en scène certains aspects de l'écriture ${ }^{(9)}$. Dans la lignée des travaux de ''ITEM $^{(10)}$, de Claudine Fabre-Cols (2004) ou encore de ceux de Claire Doquet-Lacoste (2004a), ces conventions, que nous proposons, sont réalisées par un codage manuel; très proches de ceux déjà développés pour l'oral, ils permettent d'appréhender de manière simple la richesse et la complexité des phénomènes impliqués, et le plus souvent perdus, dans le texte produit.

Nous proposons les conventions de transcriptions suivantes pour la production écrite en temps réel ( $c f$. Tableau 1 page suivante).

La sémiologie de ces conventions reste simple : quatre types de police sont pris en compte : le simple, le barrée, l'italique et le gras. Ainsi, au geste d'ajouter, qui note le texte sans variation, est jointe la police sans effet. Au geste de supprimer, est associée la police barrée plus celle de l'italique.

Voici, à titre d'illustration, des exemples de transcriptions du processus scriptural, plus précisément des événements d'écriture, de deux productions verbales écrites. La première $(3 \mathrm{~b})$ est représentative d'une pratique novice, tandis que la seconde (4b) est représentative d'une pratique experte. Toutes les deux suivent les mêmes conventions. La comparaison des deux met en évidence le rôle déterminant du déjà écrit au sein de la pratique experte. Toutes les deux sont précédées de leurs produits respectifs ${ }^{(11)}$ (3a et $\left.4 \mathrm{a}\right)$.

(9) On peut, bien évidemment, se contenter des outils d'analyse fournis par les logiciels.

(10) L'Institut des Textes et Manuscrits Modernes reste une référence pour qui s'intéresse, de prêt ou de loin, aux transcriptions des événements scripturaux. Riche d'une expérience de plus de trente ans, l'ITEM a su mettre en place toute une série de propositions de conventions (Grésillon, 1994).

(11) Ces deux textes ont été évalués conformément au CECR : le premier ( $3 a$ /scripteur C), représentatif d'une pratique débutante, a été évalué au niveau A ; le second (4a/scripteur H), représentatif d'une pratique expérimentée, a été évalué au niveau C. 


\begin{tabular}{|l|l|l|}
\hline $\begin{array}{l}\text { Conventions } \\
\text { de transcription } \\
\text { pour le déjà écrit }\end{array}$ & $\begin{array}{l}\text { Opérations effectuées } \\
\text { à la suite du déjà écrit }\end{array}$ & $\begin{array}{l}\text { Opérations effectuées } \\
\text { par retour au déjà écrit }\end{array}$ \\
\hline Ajout & Simple & Gras \\
\hline Suppression & $\begin{array}{l}\text { - Suppression immédiate } \\
\text { Barrée avec italique } \\
\text { - Suppression différée } \\
\text { Barrée sans italique }\end{array}$ & $\begin{array}{l}\text { - Suppression immédiate } \\
\text { Barrée avec itatique en gras } \\
\text { - Suppression différée } \\
\text { Barrée sans italique en gras }\end{array}$ \\
\hline Remplacement & Remplaeéremplacant & Remplacéremplacant \\
\hline Déplacement & Béplacédéplacant & Béplacédéplacant \\
\hline
\end{tabular}

Tableau 1. Conventions de transcriptions scripturales mettant en relief le déjà écrit.

\subsection{Transcription d'une pratique novice de l'écriture}

Pour respecter les habitudes liées à l'évaluation des textes en production écrite, voici le texte final produit tel qu'il est édité par le logiciel Scriplog :

(3a) Je pense qu'un ideale place à vivre était comme mon pays, Finlande. Les gens pourraient être plus gentil à mon avis. Ils pourraient être plus tolerants. Je voudrais vivre dans un petit ville, avec ma famille, mes amis et mes chats. Je vivrais dans un petit maison avec un petit jardin. Mes amis vivaient très proche de moi, et nous pourraions faire un petit rendez-vous tous les jours. Je voudrais que la ville était très, très petite, parce que je voudrais connaître tous les habitants de cette ville. Le monde autour de moi était pleine de paix, il n'y avait pas les guèrres. Tout le monde s'aimeront. Je voudrais que mes enfants d'avenir pourraient aller à l'école, qui était très petite aussi.

(Scripteur C)

Un tel texte (3a) reste conforme aux attentes du genre ; à ce titre, il est, par tradition, susceptible d'être corrigé, voire évalué. Peut-on se contenter, d'une manière paradoxale, de continuer d'affirmer qu'il s'agit de production et, en même temps, continuer de travailler à partir d'un produit ?

Ce que nous venons de voir, c'est le produit écrit, et non le processus d'écriture ! Voici donc, une tentative de représentation, de transcription de ce même processus :

(3b) Je pense qu un ideaktle place ß Đà vivre ètaitétait comme mon pays-, Finlandeme. Je pense que/A mon avis/H y a tous/Peut./-être il pourrait être un peu plus chaud ouLes gens pourraient $E$ être plus gentil à mon avis. Ils pourraineent être plus tolerab/tnts. Je vourdrais vivre dans un petit ville, avec mea famille, \#mes amis et menes chats. AousLa ville ètait trè/es/rès Je vivrais à un petit dans un petit maison quiavec un trmpetit jardin-. Mes amis vivraient très proche de moi, et nous pourrions faire un petit rendez. - vous tous les jours. Ea the voudrais que la ville était très-, très peittite, parce que je voudraias sonnaître tous keles habitants-de cette ville. ELe monde autour de moi ‘ètaitétait plein e de paix, il n'y $a$ avait pas les guèrres. Toust le monde aimeronts' aimeront. Je voudrais que mes enfants,-/pourraie dé' avenir pourraineent aller $A$ à l'école-, qui 'était tréès petite aussi. icilei,/ ils pourraient éte '/e/é les professeurs les

(Scripteur C)

Ainsi, la lecture de la transcription, aux lignes 2 et 3 , donne à voir la séquence suivante qui recoupe la deuxième phrase du texte fini : 
Je pense quel A mon avis/Il y a tous/Peut./-être il pourrait être un peu plus chaud $\theta u$ Les gens pourraient Eêtre plus gentil à mon avis

La séquence Je pense que disparaît aussitôt qu'elle est écrite, et laisse place à $A$ mon avis. Celle-ci, à son tour, est supprimée pour laisser la place à la séquence $I l y$ a tous, qui sera, à son tour, supprimée pour laisser la place à Les gens pourraient être plus gentil à mon avis (avec une suppression de $E$ pour $\hat{e}$ ).

En fait, il est très délicat de décrire, sans prendre parti, ce qui vient de se passer : les verbes utilisés (supprimer et remplacer) induisent déjà une interprétation en superposant la succession des opérations d'écriture aux événements. La similarité sémantique des séquences pourrait laisser croire que la suivante remplace la précédente sans perte.

\subsection{Transcription d'une pratique experte de l'écriture}

Voici d'abord le texte final produit (4a) :

(4a) Environnement idéal

Qu'est-ce qui me vient en tête spontanément quand on me donne la tâche de décrire un environnement idéal ? Beaucoup de verdure, peu de pollution, suffisamment d'espace pour que tout un chacun se trouve à l'aise avec soi-même et avec les autres. Le climat ? Je dois dire que les saisons distinctes et variées typiques du Nord me plaisent, etv que c'est quelque chose que je voudrais avoir dans un environnement idéal. Autrement dit, un été chaud et ensoileillé sans oublier évidemment les journées lumineuses et extrêmement longues comme en Finlande en été, un hiver blanc, avec du soleil et de la neige, et encore deux saisons de "transmission", de passage on peut dire, pour passer du froid au chaud, ou inversement : le printemps et l'automne.

S'il n'est pas possible, pour moi, d'imaginer une vie idéale sans la nature telle que je la connais dans mon pays natale, il m'est tout aussi impossible d'imaginer une existence heureuse sans la présence des enfants et des animaux. Les enfants et les animaux font partie de la nature.

J'ai toutjours vécu au bord de la mer, ou plus précisément, je vis mes hivers au bord de 1 amer proprement dite et mes étés à la campagne au bord d'un grand lac. Cette présence de l'eau dans mon environnement est quelque chose qui me manque lorsque je suis

(Scripteur H)

Puis, voici la transcription des événements d'écriture ; à la différence de la précédente, celle-ci (4b) fait apparaître, en caractère gras, des nombreux retours dans le déjà écrit :

(4b) Environnaement idéal

Quementest-ce qui me vient en tête spontanément quand on me donne la tâche d’e décrire un environnement idéal ? ${ }^{18} \mathrm{Be} \boldsymbol{t}^{1}$ au ${ }^{2}$ coup de verdure $\nabla^{1}{ }^{2}{ }^{2}$, peu de pollution, suffisamment d'espace pour que trotout un chacun se trouve \%'tà

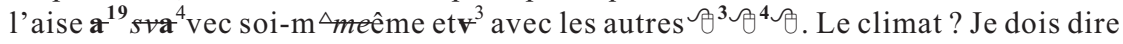
que les saisons disctinctes et variées typiques du Nord ma $\mathrm{e}^{5}$ plaisent $\boldsymbol{\vartheta}^{5} \sqrt{3}$, etv que ce'est vquelque chose que je voudrais avoir dans un $\boldsymbol{u}^{10}$ nvironnement idéal. ĐoAutrement $\mathbf{n}^{11}$ dit, un été chaud et ensoileillé aveesans oublier évidemment les journées lumineuses et ex $\mathbf{x}^{6}$ trêmement longues $\mho^{6} \hat{0}$, tacomme en Finlande en été, un hiver blanc, avec du $\mathbf{d \mathbf { s } ^ { \mathbf { 1 2 } }}$ oleil et de la natgeeiłge, et encore dtreu $\mathrm{x}^{\mathbf{1 3}}$ saistons de "transmisson", de passage on peut dire, pour passer du froid, ${ }^{14}$ au chaud ou täineinversemenrtt: le prinpntemps et l'a automne.

tene peuxS'il n'est pas possible, pour moi, d'imaginer une vie idéale sans la nature éblouissante telle que je la connais dans mon pays natale, il $\mathbf{~ m m}^{7}$ 'üe'est égatout aussi im ${ }^{8}$ possible non plus pot ${ }^{9} e^{7} \diamond^{8} \vartheta^{9}{ }^{*}$ d'imaginetri un-eviee existence ${ }^{15}$ heu- 
reuse sans la på ${ }^{16}$ résence des enfants et des animaux. $\beta^{10} \beta^{11} \beta^{12} \beta^{13} \beta^{14} \beta^{15} \beta^{16} \beta^{17}$ ?. Les enfants et les animaux font partie de la nature ausens. $\beta^{18} \beta^{19} \beta$

Foute mavie, jä'at'-J'ai toujours vécu au bord de la mer-/m, ou plus précisément, je vis mes hivers au bord de 1 amer proprenent dite et mes étés à lampagne au bord d'un grand lac. Cette présence de l'eau dans mon environnement qest quelque chose qui me manque lorsque je suis

(Scripteur $\mathrm{H}$ )

Cette transcription applique les grandes lignes précédemment décrites. L'indice numérique indique le rang de la révision effectuée par le scripteur ${ }^{(12)}$. Il est, nécessairement, accompagné soit de la représentation d'une flèche, soit de la représentation d'une souris. Il faut alors comprendre qu'à l'occasion d'une manipulation de la flèche ou de la souris, le scripteur a procédé à des ajouts ou à des suppressions : par retour dans le déjà écrit, il sera facile de retrouver où ce dernier est intervenu précisément.

Ainsi, la lecture de la transcription (ligne 2) donne à voir la séquence suivante :

$$
\text { Bet } \mathbf{u}^{1} \mathbf{u}^{2} \text { coup de verdure } \leftarrow^{1} \rightarrow^{2}
$$

La notation $\leftarrow^{1}$ est à comprendre comme la marque de la manipulation, par le scripteur, de la flèche gauche pour revenir dans son déjà écrit et y effectuer, dans le cas présent, une suppression, notée $\mathbf{t}^{1}$ avec le même indice numérique. Puis ce même scripteur, en utilisant la flèche droite, notée $\rightarrow^{2}$, pour revenir vers la droite, fera un ajout, noté $\mathbf{u}^{2}$.

Rien d'étonnant donc de voir apparaître (4b), en caractères gras, un premier énoncé suivi de 1'indice 18 ; celui-ci montre que cet énoncé, première phrase du texte produit, est en fait, le dix-huitième retour dans le déjà écrit, en phase de production.

Une telle transcription du processus d'écriture reste riche d'ouvertures. Tout d'abord, elle propose une notation de la chronologie des événements d'écriture. Ce genre de transcription prend note d'une chronologie relative des événements. Elle ne prend pas note, comme le fait le fichier d'enregistrement des événements, d'une chronologie absolue qui montre le temps écoulé (en minutes, secondes et millièmes de secondes) depuis le début de la tâche d'écriture. Elle note une chronologie des événements les uns par rapport aux autres, en attirant l'attention sur la succession des événements (Leblay, 2009).

Elle autorise, ensuite, une prise en compte du nombre exact des manipulations de la souris et des flèches à partir desquelles sont effectués les retours dans le déjà écrit ; ce qui reste une information très précieuse, puisqu'elle prépare à ce que peut être l'opération d'écriture en prenant soin de distinguer celles qui sont effectuées à la suite du déjà écrit (ajouts, suppressions, remplacements et déplacements) de celles qui sont effectuées par retour dans le déjà écrit ${ }^{(13)}$ (ajouts, suppressions, remplacements et déplacements).

Enfin et surtout, il convient de remarquer combien le texte final, le produit, est peu ressemblant à la production, à l'écriture de ce produit (Leblay, 2007b). Ainsi,

(12) L'idée de cet indice numérique est empruntée à Ann Matsuhashi (1981, 1987); voir aussi l'article d'Eva Lindgren et de Kirk P. H. Sullivan (2006) relatif aux processus de révision en temps réel.

(13) Il serait même possible de déceler, dans 4b, une suppression d'un autre type (ligne 1: Queme vionet'), puisque le scripteur procède à une suppression à la suite d'un déjà écrit (inscription de Qu-) lui-même produit par retour dans du déjà écrit. 
la première phrase du texte fini (4a) a été rédigée en fin d'écriture (4b) malgré la mention explicite, dans la première phrase (4a), de l'adverbe spontanément. Apparaît ici toute la stratégie du scripteur, toute sa ruse à prétendre du spontané, là où il y a du calculé, de l'artifice. Cette écriture se situe, pour reprendre les mots d'Irène Fenoglio, « du côté de la maîtrise », cette écriture est «jouée » $(2001,178)$.

\section{Exploitation génétique}

Dès lors, on peut essayer de caractériser l'expertise d'une écriture et d'en montrer certaines régularités ${ }^{(14)}$. Ainsi, l'opposition entre produit et production permet de repenser un double cadre, théorique et pratique, dans lequel la notion du déjà écrit ${ }^{(15)}$ fait saillie.

\subsection{Production vs produit}

Contrairement à ce que 1'on pourrait attendre, le produit n'est pas l'image fidèle de la production : chercher à comprendre le processus d'écriture à partir du produit est peut-être illusoire. Il est clair que le produit final est plus réduit, plus court, plus coupé que la production ${ }^{(16)}$. A comparer les productions et les produits des écritures, expertes ou novices, il ressort que, dans les deux cas, le nombre d'événements en production est bien supérieur à celui des événements pris en considération dans l'établissement du texte final ${ }^{(17)}$ (tableau 2).

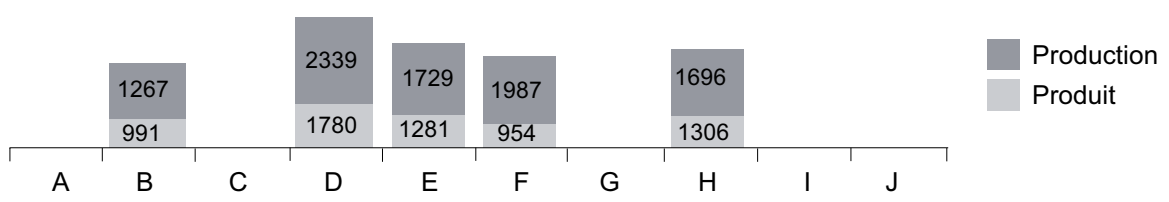

Tableau 2. Corrélation entre le nombre total d'événements en production et le nombre d'événements restant dans le produit final (écriture expérimentée).

On peut s'interroger alors sur le bien fondé, pour un enseignant, de laisser de côté autant d'éléments offerts par la production pour se contenter de si peu d'informations données par le produit sur la manière d'écrire de tel ou tel scripteur.

Paradoxalement, les habitudes prises, tant par les enseignants que par les apprenants, font que tout est orienté vers le texte; lequel est senti comme l'unique objet d'attention et de description.

(14) Pour une revue de détails sur le développement de l'expertise en production écrite, voir l'ouvrage de Denis Alamargot et de Lucile Chanquoy (2001, ch. 6 pp. 185-218).

(15) Voir, pour plus de détails, Lacoste (2004) et Leblay $(2009,2007 a)$.

(16) Michel Arrivé $(2007,101)$ fait référence à la notion d'anéantissement total du texte ou d'une partie du texte dont il ne reste rien. Cette notion rentre en concurrence directe avec l'opération d'écriture de suppression, mal adaptée pour rendre l'idée de suppression pure et simple d'un élément dont il ne resterait rien.

(17) Les écritures novices présentent la même variation entre produit et production, soit : Scripteur A (943/558), Scripteur C (1253/698), Scripteur G (1106/618), Scripteur I (1318/638) et Scripteur J (1885/1205), où le premier chiffre représente la quantité d'événements en production, et le second, la quantité d'événements restant dans le produit fini, c'est-à-dire dans le texte. 


\subsection{Enseigner les retours dans le déjà écrit}

Au sein de nombreuses activités de production, une pratique se distingue : celle d'apprendre à reconsidérer ce qui est déjà écrit. Pourquoi ? Parce qu'elle indique la route simple vers une écriture plus expérimentée (tableau 3).

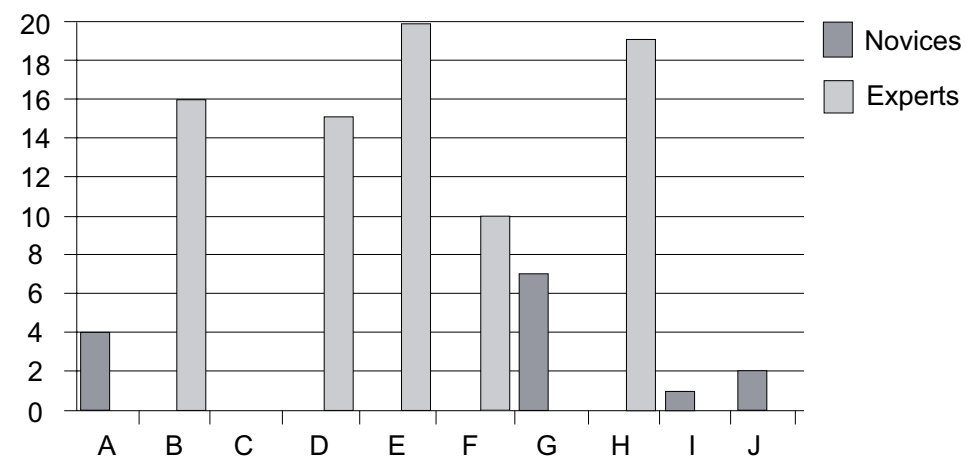

Tableau 3. Nombre des retours dans le déjà écrit. Ecritures débutantes (scripteurs A, C, G, I et J) et écritures expérimentées (scripteurs B, D, E, F et H).

Les pratiques expertes se distinguent des pratiques novices, comme on pouvait le supposer, par le nombre de retours dans le déjà écrit. Ainsi, dans le modeste corpus présent, le nombre moyen de retour dans le déjà écrit est, pour les pratiques expertes, de 16 , tandis qu'il est de 2.8 pour les pratiques novices.

Il est donc possible de comptabiliser le nombre de retours dans le déjà écritet de savoir où exactement ceux-ci ont pris place lors de la production, mais il est surtout possible de s'apercevoir que certaines écritures n'ont donné lieu à aucun retour (exemple $3 b$ ). Comment alors ne pas prendre acte de ces stratégies par défaut? Comment ne pas enseigner, en prenant le scripteur à témoin de sa propre production, le lien explicite existant entre les choix qu'il vient de faire (de ne pas revenir vs revenir dans son écriture) et les résultats qualitatifs auxquels il parvient (produit non acceptable $v s$ acceptable) ? Il ne s'agit pas seulement d'apprendre à relire son (texte) écrit, comme souvent on le dit, mais bien d'apprendre à relire son écriture pour y revenir, y faire les modifications nécessaires, pour y réécrire.

Ce genre de remarques nous amène directement à nous intéresser, dans la ligne des travaux réalisés à l'ITEM, au dialogue incessant entre le scripteur et son déjà écrit. Depuis les années 1983-84, depuis les travaux fondateurs de Claudine Fabre qui réalisa le lien entre didactique de l'écriture et critique génétique, il est dorénavant possible, non seulement d'observer des événements d'écriture, mais aussi de considérer les productions d'apprenant depuis les opérations d'écriture.

\subsection{Les opérations d'écriture}

Contrairement à l'événement d'écriture, l'opération d'écriture est une interprétation : elle cherche à rendre compréhensible ce qui est dense, complexe ou ambigu. Il s'agit d'une lecture critique d'un fait de langue. Celle-ci relève d'un sens personnel, parmi d'autres possibles qui n'apparaissent pas de manière évidente. Là encore, il s'agit de partir d'un des constats, sur lequel repose la critique génétique, qui veut que tout écrit s'élabore dans le temps. 


\subsubsection{Les opérations d'écriture effectuées à la suite du déjà écrit}

Dans une approche inductive, ces deux tableaux (4a et $4 b$ ) listent les formes transcrites obtenues à partir du fichier d'enregistrement et de la traduction linéaire des événements. Toutes sont chronologiquement indexées sous la forme ${ }^{<s e-}$ condes.millièmes de secondes>; à ce premier indice temporel, est ajouté un second indice $<\mathrm{x}>$, séparé du premier par les deux points, qui correspond au nombre d'événements pris en compte dans le geste élémentaire de suppression-ajout ${ }^{(18)}$ (voir le fichier d'enregistrement et la traduction linéaire). Le tableau $4 \mathrm{a}$ offre donc toutes les occurrences produites à la suite du déjà écrit par un scripteur expérimenté $(\mathrm{H})$.

\begin{tabular}{|c|c|c|c|c|}
\hline $\begin{array}{l}\text { Transcriptions } \\
\text { linéaires } \\
(32) \\
\text { Scripteur H }\end{array}$ & \multicolumn{4}{|c|}{ 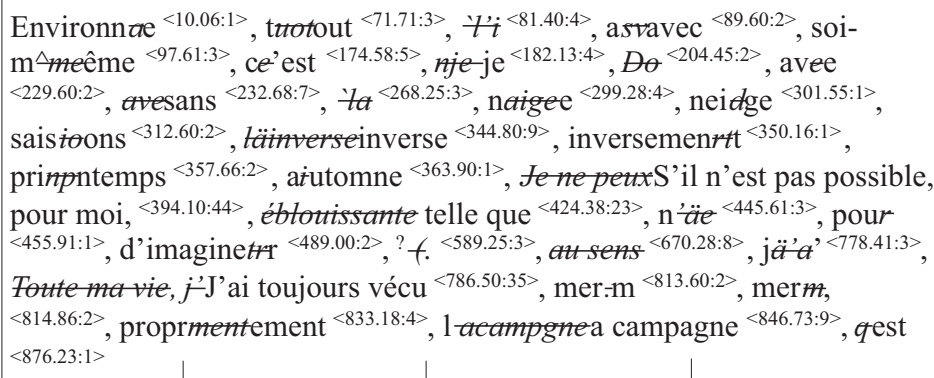 } \\
\hline \multirow{2}{*}{$\begin{array}{l}\text { Opérations } \\
\text { d'écriture }\end{array}$} & & Suppressions & Remplacements & Déplacements \\
\hline & & & & \\
\hline
\end{tabular}

Tableau $4 a$. Les opérations d'écriture à la suite du déjà écrit (correspondantes à la transcription de 4 b).

Comment alors interpréter ces opérations d'écriture ? Sont-elles des suppressions, des remplacements ou bien des déplacements?

Par exemple, l'opération réalisée en "avesans », est-elle interprétable en termes de suppression de « ave », qui serait suivie de celle d'un ajout de « sans »? Ou bien est-elle à interpréter en tant qu'opération de remplacement (« avec » remplacé par «sans ») ? Peut-on dire qu'un signifiant disparaît totalement au profit d'un autre, ou bien qu'un signifiant inscrit, puis effacé, permet l'inscription d'un second plus satisfaisant ? Cette notation, «avesans », plaide, sans ambiguïté pour 1'opération de remplacement, en comptabilisant sept événements.

Ce genre de matériel est technique et lourd, mais il est également riche. En listant puis en essayant de classer ces formes, l'enseignant apprend à voir autrement, il apprend à interpréter des formes précises. Il ne s'agit plus de corriger telle ou telle faute, mais bien d'interpréter des modifications, des « ruminations » (Fenoglio, 2007) : plus précisément encore, il s'agit d'interpréter des productions en nommant explicitement telle opération ou telle opération. Ainsi, l'opération de remplacement permet de décrire une tentative première, provisoire, qui cherche à faire exister une forme seconde, plus permanente, plus proche de ce qui veut être dit. Cette part ajoutée, immédiatement successive, ressemble à sa part supprimée. Le scripteur tente, ose une première forme (un premier signifiant) pour pouvoir la reprendre immédiatement sur le mode de l'analogie afin d'obtenir une autre forme

(18) Ces gestes élémentaires d'ajout ou de suppression (noté $<$ CR $>$ dans le fichier d'enregistrement) ne sont pas à confondre avec les opérations d'ajout et de suppression.

(19) La description de l'ajout à la suite du déjà écrit, ou inscription, n'est pas prise en compte dans ce tableau; il est d'ailleurs transcrit par une police sans effet ( $3 \mathrm{~b}$ et $4 \mathrm{~b})$. 
peut-être plus satisfaisante. Un signifiant sera posé momentanément et rapidement un autre viendra prendre sa place. C'est précisément dans ce jeu de signifiants successifs, que réside toute la dimension temporelle de l'écriture.

\subsubsection{Les opérations effectuées par retour dans le déjà écrit}

Dans le même ordre d'idée, il est nécessaire de présenter les opérations s'inscrivant par retours dans le déjà écrit. Ces dernières, conformément aux conventions citées précédemment, font toutes intervenir la police de caractères gras et restent très représentatives d'une pratique expérimentée de l'écriture.

\begin{tabular}{|c|c|c|c|c|}
\hline $\begin{array}{l}\text { Transcriptions } \\
\text { linéaires } \\
(21) \\
\text { Scripteur? }\end{array}$ & \multicolumn{4}{|c|}{ 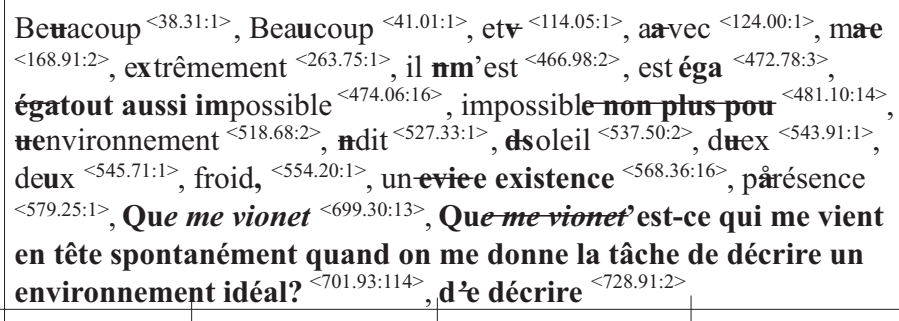 } \\
\hline $\begin{array}{l}\text { Opérations } \\
\text { d'écriture }\end{array}$ & Ajouts & Suppressions & Remplacements & Déplacements \\
\hline
\end{tabular}

Tableau $4 b$. Les opérations d'écriture par retour dans le déjà écrit (correspondantes à la transcription $4 \mathrm{~b})$.

A isoler ainsi ces substitutions pour ce qu'elles sont, c'est apprendre à se focaliser sur ce qui n'apparaît jamais dans une copie « au propre », c'est apprendre et enseigner le geste prépondérant de revenir sur son déjà écrit dans l'écriture. Comme le souligne Almuth Grésillon (Authier-Revuz et Grésillon 2004, 9), « il n'y a pas d'écriture sans réécriture [...], pas plus qu'il n'y a de formulation sans reformulation $\gg$.

Il s'agit d'apprendre à écrire à partir de différentes sources mettant en scène le temps de 1'écriture, mais aussi, d'apprendre à écrire à partir d'une écriture saisie par son propre temps : impossible d'échapper à cette double direction. A chacun de se rendre compte de la variété et de la pertinence de ces retours sur le déjà écrit, de ces retours sur soi que livrent d'authentiques productions, bien loin des produits prétendument «bons » ou « mauvais » habituels. Les productions présentes offrent, du point de vue génétique, les mêmes caractéristiques que des productions de langue maternelle. Car, s'il est vrai que la « dynamique énonciative, en tant que besoin expressif du sujet, n'a pas d'âge » (Fabre-Cols, 2004), elle n'a pas non plus d'origine linguistique.

\section{Pour finir}

Au risque de nous répéter, il est maintenant possible d'apprendre à considérer un corpus de production verbale écrite à partir d'un nombre de secondes, minutes et d'heures, et non pas seulement à partir d'un nombre de pages données.

Les événements d'écriture ainsi que les opérations d'écriture associés aux mouvements du curseur ou équivalents (flèches et pavé tactile), sont des invariants processuels, ou opérationnels, auxquels aucun scripteur n'échappe dans sa production écrite. Ces invariants se situent en deçà de pratiques prétendument bonnes 
ou mauvaises, en deçà de pratiques expertes, ou novices, pathologiques ou déficientes, que celles-ci soient écrites en langues maternelle, étrangère ou seconde.

Les scripteurs débutants et expérimentés ont donc, à l'image de tout scripteur, en partage, au-delà des événements d'écriture, la manipulation des opérations d'écriture. Ce qui semble les différencier est la manière dont les scripteurs expérimentés se positionnent face au texte déjà écrit, c'est-à-dire face au volume textuel en mouvement, en aval de la pensée, construite sur les gestes d'ajouts et suppressions, eux-mêmes interprétés en opérations d'ajouts, de suppressions, de remplacements et de déplacements. Accepter d'écrire, c'est accepter cette confrontation avec soi-même, avec son lot de reprises, de reformulations. A partir d'un modeste corpus comme celui-ci, il s'avère déjà qu'un scripteur expérimenté reviendra (bien) plus souvent dans son déjà écrit pour, principalement, y effectuer des ajouts et des remplacements; ces opérations correspondent à des profils d'écriture et non à des profils d'individus (Rinck, 2007).

La didactique de la production verbale écrite, de l'écriture, replacée dans sa dimension intime, dans sa dimension temporelle, se voit renforcée par la démarche de la critique génétique pour laquelle, il ne s'agit pas de décrire et d'interpréter des successions d'opérations allant irrémédiablement d'un état de moindre qualité vers un état de meilleure qualité, mais allant seulement vers un état différent. L'idée sous-jacente à la démarche génétique n'est pas que le retour dans le déjà écrit est la garantie systématique d'un meilleur produit ; mais à regarder de près, c'est bien en reprenant son écriture, en ne cessant de la reprendre, en y revenant, qu'il est possible de prétendre aller vers plus de satisfaction, vers une écriture plus satisfaisante pour le sujet qui apprend à se faire face, dans ses retours sur soi, remplis d'équivoques.

Ce travail plaide ouvertement pour une prise en compte du temps de l'écriture, et de rôles redéfinis de l'apprenant-scripteur et de son enseignant. Sans chercher à savoir lequel des deux occupe le centre et lequel la périphérie, il suffit de dire que les deux ont leur place : à l'enseignant d'avoir l'initiative de proposer des outils contemporains (ordinateurs certes, mais aussi logiciels d'enregistrement), des documents visuels (à partir des linéarisations et transcriptions du processus d'écriture) pour proposer des approches différenciées (ne serait-ce qu'en demandant aux apprenants de s'enregistrer, de se transcrire et de s'observer); à l'apprenant de saisir l'occasion qui lui est offerte par son enseignant, de s'observer et d'interpréter avec lui, dans une démarche collaborative, à partir de transcriptions, son propre itinéraire.

\section{Références bibliographiques}

Alamargot, Denis et ChanQuOY, Lucile (2001) : Through the Models of Writing. Studies in Writing, Dordrecht, Boston, London, Kluwer Academic Publishers.

ARRIVÉ, Michel (2007) : «Le pied de la lettre», in Irène Fenoglio (Ed.) L'écriture et le souci de la langue, Louvain la Neuve, Academia-Bruylant, 93-112.

AUTHIER-REVUZ, Jacqueline \& GRÉSILlON, Almuth (2004) : « La réécriture. Questions théoriques », Le français d'aujourd'hui 144, 9-17.

BLANCHE-BENVÉNISTE, Claire (2000) : Approches de la langue parlée en français, Paris, Ophrys. 
CADRE EUROPÉEN COMMUN DE RÉFÉRENCE : APPRENDRE, ENSEIGNER, ÉVALUER (2001), Conseil de l'Europe, Division des politiques linguistiques, Paris, Didier.

Doquet-L ACOSTE, Claire (2004a) : Etude génétique de l'écriture d'élèves de Cours Moyen 2, année 1995-96, Thèse de Doctorat de Sciences du Langage soutenue à l’Université de Paris 3 le 17 juin 2003, Lille : Atelier National de Reproduction des Thèses.

— (2004b) : «Indices et traces de l'activité métadiscursive des scripteurs », Le français aujourd'hui 144, 33-41.

FABRE-COLS, Claudine (2004) : «Les brouillons et l'école : ce qu'a changé la critique génétique », Le français aujourd'hui 144, 18-24.

FENOGLIO, Irène (2009) : «Les notes de travail d'Emile Benveniste : où la pensée théorique naît via son énonciation », Langage \& Société 127, 23-49.

- (2001) : «Les événements d'énonciation : Focaliseurs d'interprétation pyschanalytique, matériau pertinent de l'analyse linguistique », in Michel Arrivé et Claudine Normand (Eds.) Linguistique et psychanalyse, Colloque international de Cerisy-La-Salle, Septembre 1998, 167-184.

GRÉSILlON, Almuth (1994) : Eléments de critique génétique, Paris, PUF.

LEBLAY, Christophe (2009) : «La question du « déjà écrit» dans le processus d'écriture observé en temps réel. Une contribution de la génétique à la didactique », in J.-M. Adam et I. Fenoglio (Eds.) Approches nouvelles, champs neufs, innovations techniques : une actualisation de la génétique du texte, Modèles linguistiques, (A paraître).

— (2007a) : «L'avant-texte comme texte sur le vif. Analyse génétique d'opérations d'écriture en temps réel », Langue française 155, 95-108.

— (2007b) : «L'avant-texte: le texte en émoi », in Juhani Härmä, Ulla Tuomarla et al. (Eds.) Actes du XXIXème Colloque International de Linguistique Fonctionnelle, Département des Langues Romanes de l'Université de Helsinki 18, 141 146.

Lindgren, Eva et S Ullivan, Kirk P.H. (2006) : «Analysing Online Revision », in K. P. H. Sullivan et E. Lindgren (Eds.) Computer Keystroke Logging and Writing, Amsterdam, Elsevier, 157-188.

MAtsuhashi, Ann (1987) : «Revising the plan and altering the text», in Ann Matsuhashi (Ed.) Writing in real time, Norwood, New Jersey, Ablex Publishing Corporation, 197-223.

— (1981) : «Pausing and planning: The tempo of written discourse production », Research in the Teaching of English 15(2), 113-134.

RINCK, Fanny (2007) : «Styles d'auteur et singularité des textes. Approches stylométrique du genre de l'article en linguistique », Pratiques 135-136, 119-136.

STRÖMQVIST, Sven (2006a) : «Une approche expérimentale du processus d'écriture : l'enregistrement de la frappe au clavier ", Genesis 27, 45-57.

STRÖMQVIST, Sven et al. (2006b) : «What Keystroke Logging can Reveal about Writing », in G. Rijlaarsdam (Series Ed.) and Kirk P.H. Sullivan \& Eva Lindgren (Vol. Eds.), Studies in Writing, Vol. 18, Computer Keystroke Logging : Methods and Applications, Oxford, Elsevier, 45-71. 